\title{
Model of an openable Faraday-effect hybrid-current optical transducer based on a square-shaped structure with internal mirror
}

\author{
J. L. Arce-Diego, R. López-Ruisánchez, J. M. López-Higuera, and M. A. Muriel
}

\begin{abstract}
A new type of clamp-on magneto-optical current transducer based on the Faraday effect is presented. It is openable, hybrid, and square-shaped, and it has an internal mirror that allows the current sensitivity to improve and the effects of the optical activity and temperature-dependent linear birefringence to be rejected. The design and the simulated behavior of the proposed device are described and compared with the conventional (no mirror) transducer. (C) 1997 Optical Society of America
\end{abstract}

A magneto-optical current sensor (MOCS) is an optical device in which the polarization azimuth of a linearly polarized light beam propagating inside a dielectric is rotated under the influence of a magnetic field generated by the electrical current to be measured. This kind of sensor has the advantages of electrical insulation, high electromagnetic immunity, and a simpler structure when compared with conventional transducers. All-fiber and hybrid MOCS technologies are currently used. In the former, an optical fiber itself acts as a sensing element, forming a coil-shaped optical path around the conductor. However, it has important disadvantages owing to its small Verdet constant and relatively high linear birefringence. In the hybrid MOCS the optical path is embedded in a magneto-optical material, and the fibers are used as a communication channel between the transducer and the optoelectronic unit. The advantages of this kind of sensor include its higher Verdet constant and the possibility of removal without cutting the electrical conductor. However, there are several drawbacks related to the materials currently used, including optical activity, linear birefringence, and temperature dependence.

Several setups have been presented. Triangular ${ }^{1}$

J. L. Arce-Diego, R. López-Ruisánchez, and J. M. López-Higuera are with the Grupo de Ingeniería Fotónica, Dto. Tecnología Electrónica, Ingeniería de Sistemas y Automatica, Universidad de Cantabria, 39005 Santander, Spain. M. A. Muriel is with the Dto. Tecnología Fotónica, Universidad Politécnica de Madrid, 28040 Madrid, Spain.

Received 10 June 1997.

0003-6935/97/256242-04\$10.00/0

(C) 1997 Optical Society of America and circular ${ }^{2}$ topologies are based on critical angle reflections to avoid changes in the linear state of polarization of the incident light. But precise designs are required because variations in the reflection angle cause disturbance of the linear polarization. Note that this critical angle depends on the refractive index, which is a function of the wavelength.

The proposed optical transducer has a squareshaped geometry (Fig. 1). To allow the transducer to be assembled around an existing conductor, it can be broken into two symmetrical parts. This transducer uses dual-quadrature reflections to preserve the linear polarization of light and to avoid the need of critical angle reflections. The inclusion of a metallic mirror at the end of the optical path improves current sensitivity thanks to the nonreciprocity of the Faraday effect, and it minimizes the effects of both linear birefringence and optical activity (reciprocal effect) with respect to the conventional squareshaped transducer. ${ }^{3}$

It is well known that certain materials act as polarization rotators when placed in a magnetic field. The orientation of linearly polarized light propagating in such materials suffers a rotation under the influence of that field. This rotation is known as the Faraday effect, which can be described as

$$
F=V \cdot B,
$$

where $B$ is the component of the magnetic flux density in the direction of light propagation and $V$ is the Verdet constant. Materials such as $\mathrm{Y}_{2} \mathrm{Fe}_{5} \mathrm{O}_{12}$ (YIG) and $\mathrm{Bi}_{12} \mathrm{SiO}_{20}$ (BSO) are currently used. YIG is a ferromagnetic material with a high Verdet constant, but it has several drawbacks, namely, its strong tem- 
perature dependence, saturation, and hysteresis effects together with the fact that its magneto-optical properties have geometric structure dependence. BSO crystals are diamagnetic materials with a high Verdet constant, which has little temperature dependence. ${ }^{4}$ However, BSO has optical activity, so that the current sensitivity is slightly attenuated.

The operation principles of the optical transducer are described below. For a current passing through the conductor, the component $B$ can be described as

$$
B=\mu \cdot I /(2 \pi \cdot r),
$$

where $\mu$ is the magnetic permeability of BSO (approximately equal to that in vacuum), $I$ is the electrical current to be measured, and $r$ is the distance to the conductor.

Using Jones calculus, one can obtain the output optical field for the proposed transducer topology of Fig. 1:

$$
\begin{aligned}
\mathbf{E}_{\text {out }}= & \mathscr{P}(\alpha) \cdot\left(\mathscr{F}_{B 1} \cdot \mathscr{R}_{\perp} \cdot \mathscr{F}_{B 1}{ }^{\prime} \cdot \mathscr{R}_{\|} \cdot \mathscr{F}_{B 2} \cdot \mathscr{R}_{\perp}\right. \\
& \left.\cdot \mathscr{F}_{B 2}{ }^{\prime} \cdot \mathscr{R}_{\|} \cdot \mathscr{F}_{B 3} \cdot \mathscr{R}_{\perp} \cdot \mathscr{F}_{B 3}{ }^{\prime} \cdot \mathscr{R}_{\|} \cdot \mathscr{F}_{B 4}\right) \cdot \mathscr{R}_{\mathrm{MET}} \\
& \cdot\left(\mathscr{F}_{A 4} \cdot \mathscr{R}_{\perp} \cdot \mathscr{F}_{A 3}{ }^{\prime} \cdot \mathscr{R}_{\|} \cdot \mathscr{F}_{A 3} \cdot \mathscr{R}_{\perp} \cdot \mathscr{F}_{A 2}{ }^{\prime} \cdot \mathscr{R}_{\|}\right. \\
& \left.\cdot \mathscr{F}_{A 2} \cdot \mathscr{R}_{\perp} \cdot \mathscr{F}_{A 1}{ }^{\prime} \cdot \mathscr{R}_{\|} \cdot \mathscr{F}_{A 1}\right) \cdot \mathbf{E}_{\mathrm{in}},
\end{aligned}
$$

where $\mathscr{P}(\alpha)$ is the Jones matrix for a polarizer whose transmission axis forms an angle $\alpha$ with the $x$ axis:

$$
\mathscr{P}(\alpha)=\left(\begin{array}{cc}
\cos ^{2} \alpha & \sin \alpha \cos \alpha \\
\sin \alpha \cos \alpha & \sin ^{2} \alpha
\end{array}\right) .
$$

$F_{F}$ is the Jones matrix for a magneto-optical crystal. The $A 1$. . A 44 subscripts refer to the four sides of the forward optical path, and the $B 1 \ldots B 4$ subscripts refer to the backward ones:

$$
p=\left[\beta^{2}+4(G+F)^{2}\right]^{1 / 2},
$$

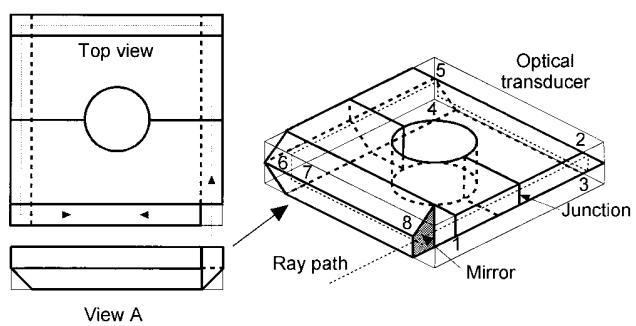$$
\mathscr{F}_{A}(F, G, \beta)=\left[\begin{array}{cc}
\cos \left(\frac{p L}{2}\right)+j \frac{\beta}{p} \sin \left(\frac{p L}{2}\right) \cos (2 \phi) & j \frac{\beta}{p} \sin \left(\frac{p L}{2}\right) \sin (2 \phi)+\frac{2(G+F)}{p} \sin \left(\frac{p L}{2}\right) \\
j \frac{\beta}{p} \operatorname{sen}\left(\frac{p L}{2}\right) \operatorname{sen}(2 \phi)-\frac{2(G+F)}{p} \operatorname{sen}\left(\frac{p L}{2}\right) & \cos \left(\frac{p L}{2}\right)-j \frac{\beta}{p} \operatorname{sen}\left(\frac{p L}{2}\right) \cos (2 \phi)
\end{array}\right],
$$

where $F$ is the Faraday effect induced by the current, $G$ is the BSO optical activity, $\beta$ is the total linear birefringence per unit length, $L$ is the side length, and $\phi$ is the angle made by the characteristic direction of the linear birefringence with the $x$ axis. To obtain the Faraday matrix with the $B 1 \ldots B 4$ subscripts, $F$ must be substituted by $-F$ in Eqs. (5) and (6) to express the nonreciprocity of the Faraday effect. $\mathscr{F}^{\prime}$ is the matrix that corresponds to trajectories between quadrature reflections, and it is equal to the unity matrix because the magnetic field is transverse to the optical path. The reflection matrix (ideal mirror) is

$$
\mathscr{R}_{\mathrm{MET}}=\left(\begin{array}{cc}
-1 & 0 \\
0 & 1
\end{array}\right) .
$$

$\mathscr{R}_{\perp}$ and $\mathscr{R}_{\|}$are the matrices for total internal reflections at the transducer corners:

$$
\mathscr{R}_{\perp}=\left(\begin{array}{cc}
\delta_{\perp} & 0 \\
0 & \delta_{\|}
\end{array}\right), \quad \mathscr{R}_{\|}=\left(\begin{array}{cc}
\delta_{\|} & 0 \\
0 & \delta_{\perp}
\end{array}\right),
$$

where $\delta_{\perp}$ and $\delta_{\|}$are the phase jumps when the field is either perpendicular or parallel to the plane of incidence. They are both of the type $\exp (j a)$, so that they have a unity modulus.

The square shape is based on dual-quadrature reflections, ${ }^{5}$ so that reflections do not need to be at a critical angle and they do not alter the state of polarization of incident light. The total internal reflection produces a phase delay between the two orthogonal components of the incident field:

$$
\mathbf{E}_{\text {out }}=\left(\begin{array}{cc}
\delta_{\perp} & 0 \\
0 & \delta_{\|}
\end{array}\right) \mathbf{E}_{\text {in }}
$$

In the second reflection, the parallel component is
Fig. 1. Optical bulk current transducer with internal mirror. The topology allows the transducer to be separated in two symmetrical pieces: 1, input point; $2-7$, total internal reflection points in the transducer corners; 8 , reflection at the mirror. now the perpendicular one and vice versa, so that the total output field after the quadrature reflections is

$$
\mathbf{E}_{\text {out }}=\delta_{\|} \delta_{\perp}\left(\begin{array}{ll}
1 & 0 \\
0 & 1
\end{array}\right) \mathbf{E}_{\text {in }} .
$$

As can be seen, the input state of polarization is maintained. The mirror reflects the incident light, so that the light travels backward along the optical path to the emitter. To launch and gather the optical signal according to the requirements, the polarizing unit shown in Fig. 2 is used. The input light, which comes from a LED through a multimode fiber, 


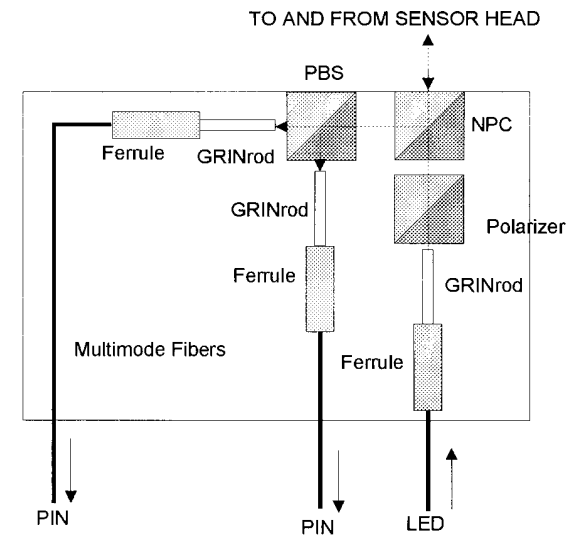

Fig. 2. Polarizing unit: GRIN rod, gradient-index rod.

is converted into linearly polarized light. This light passes through a nonpolarizing cube (NPC) and enters the transducer. A polarizing beam splitter (PBS) is used to separate the output light into its two orthogonal states. The PBS is rotated $\pi / 4$ with respect to the input polarizer. These two signals are sent to two PIN photodetectors, by using multimode fibers, to obtain intensities $I_{1}$ and $I_{2}$. When the classical method is used to avoid the intensity fluctuations of the source, the output signal is

$$
\begin{aligned}
S(F, G)= & \frac{I_{1}-I_{2}}{I_{1}+I_{2}}=-2\left[\frac{2(G+F)}{p} \cos \left(\frac{q L_{t}}{2}\right) \sin \left(\frac{p L_{t}}{2}\right)\right. \\
& \left.-\frac{2(G-F)}{q} \sin \left(\frac{q L_{t}}{2}\right) \cos \left(\frac{p L_{t}}{2}\right)\right] \\
& \times\left[\frac{\beta^{2}-4 G^{2}+4 F^{2}}{p q} \sin \left(\frac{q L_{t}}{2}\right) \sin \left(\frac{p L_{t}}{2}\right)\right. \\
& \left.-\cos \left(\frac{q L_{t}}{2}\right) \cos \left(\frac{p L_{t}}{2}\right)\right] \\
& -\left(\left\{\beta \left[\frac{1}{p} \cos \left(\frac{q L_{t}}{2}\right) \sin \left(\frac{p L_{t}}{2}\right)\right.\right.\right. \\
& \left.\left.+\frac{1}{q} \sin \left(\frac{q L_{t}}{2}\right) \cos \left(\frac{p L_{t}}{2}\right)\right]\right\}^{2} \\
& \left.\left.-\left[2 G \frac{2 \beta}{p q} \sin \left(\frac{q L_{t}}{2}\right) \sin \left(\frac{p L_{t}}{2}\right)\right]\right]^{2}\right),
\end{aligned}
$$

where $q$ is obtained by substituting $F$ by $-F$ in Eq. (6). If $G \gg \beta$, the output can be approximated by

$$
S(F) \approx \sin \left(4 F L_{t}\right) .
$$

This approach, when compared with the real response, gives the following error:

$$
\text { error }=[S(F, G, \beta)-S(F)] / S(F) \times 100 \% \text {. }
$$

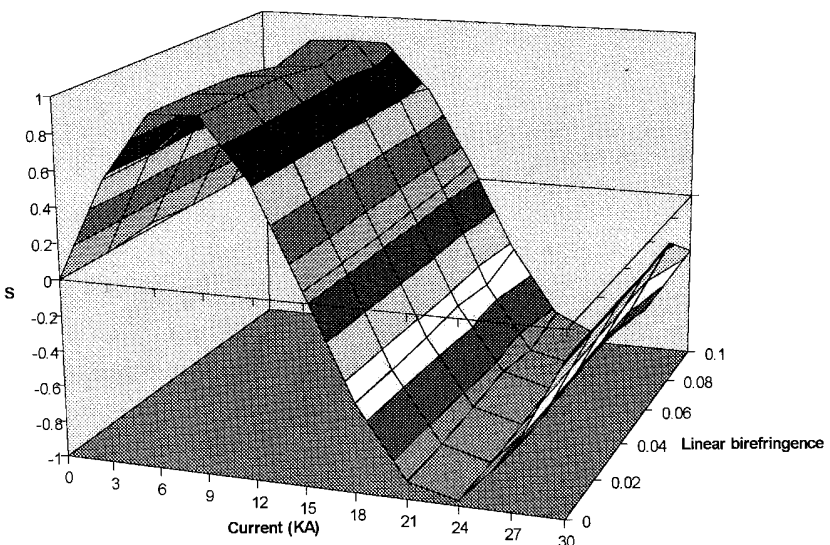

Fig. 3. Output signal for the improved transducer as a function of current and linear birefringence.

The response of the proposed transducer can be compared with the response of the square-shaped transducer with no mirror:

$$
S(F)=\left(\frac{2 F}{p}\right) \sin \left(p L_{t}\right)-\left(\frac{\beta}{p}\right)^{2} \sin ^{2}\left(\frac{p L_{t}}{2}\right),
$$

which could be approximated by

$$
S(F) \approx \sin \left(2 F L_{t}\right),
$$

when the linear birefringence $\beta$ approaches 0 . Thus, compared with Eq. (12), this transducer produces half of the rotation of the response, as would be expected since the optical path is twice as long in that case.

We carried out simulations for an optical wavelength of $830 \mathrm{~nm}$, using a transducer with a $100-\mathrm{mm}$ side length and a total length $L_{t}$ of $400 \mathrm{~mm}$. This relates to a BSO Verdet constant $V$, and optical activity of $3.2 \times 10^{-2} \mathrm{rad} / \mathrm{mm} \mathrm{T}$ and $0.192 \mathrm{rad} / \mathrm{mm}$, respectively.

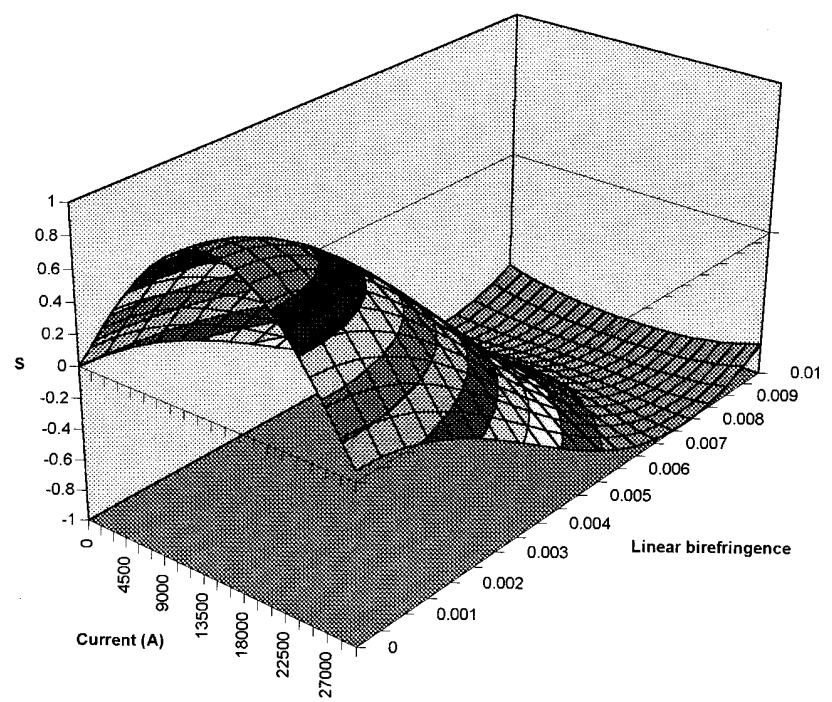

Fig. 4. Conventional square-shaped transducer response as a function of current and linear birefringence. 
The output signal for the proposed MOCS is shown in Fig. 3 as a function of the current and the linear birefringence. The current range is from 0 to $30 \mathrm{kA}$, and the maximum linear birefringence is assumed to be $0.01 \mathrm{rad} / \mathrm{mm}$. This value corresponds to an average-quality BSO crystal at $80{ }^{\circ} \mathrm{C}$, so that a range of $0-0.01$ simulates a temperature change of approximately $100{ }^{\circ} \mathrm{C}$. Figure 3 shows that the ideal sinusoidal response remains for this range, and it can be compared with the conventional (no mirror) squareshaped setup response [Eq. (14), Fig. 4]. This is strongly dependent on linear birefringence, and the sinusoidal response disappears quickly when $\beta$ is nonzero. The figure also shows that the current sensitivity (angle of rotation per unit current) for this conventional transducer is half of the sensitivity of the proposed one. The error for a BSO transducer computed by using Eq. (13) increases for small currents because the Faraday effect is smaller than the effects of optical activity and linear birefringence.

In conclusion, a new magneto-optical current transducer that uses a BSO crystal with an internal mirror has been presented. The mirror causes an improvement in the current sensitivity, and it mini- mizes the effect of both the linear birefringence and the optical activity. The temperature dependence in the new transducer is determined by the variation of the BSO Verdet constant.

This research was supported by the Spanish Ministry of Education and Science through the Comision Interministerial de Ciencia y Tecnología, project TIC95-0631-C04-01. The authors thank D. Pereda for computer simulations.

\section{References}

1. B. C. B. Chu, Y. N. Ning, and D. A. Jackson, "Faraday current sensor that uses a triangular shaped bulk-optic sensing element," Opt. Lett. 17, 1167-1169 (1992).

2. Y. N. Ning, B. C. B. Chu, and D. A. Jackson, "Miniature Faraday current sensor based on multiple critical angle reflections in a bulk-optic ring," Opt. Lett. 16, 1996-1998 (1991).

3. M. Kanoi, G. Takahashi, T. Sato, M. Higaki, E. Mori, and K. Okumura, "Optical voltage and current measuring system for electric power systems," IEEE Trans. Power Del. PWRD-1, 91-97 (1986).

4. C. M. M. van den Tempel, "Model of a new temperaturecompensated optical current sensor using $\mathrm{Bi}_{12} \mathrm{SiO}_{20}$, Appl. Opt. 32, 4869-4874 (1993).

5. M. Born and E. Wolf, Principles of Optics, 5th ed. (Pergamon, London, 1975), p. 47. 